\title{
Diferencias entre hombres y mujeres en el significado psicológico de las prácticas de socialización sexual fraternas
}

\section{Differences between men and women regarding psychological meaning of sexual socialization practices among siblings}

\author{
David Javier Enríquez Negrete ${ }^{1}$ \\ Ricardo Sánchez Medina ${ }^{2}$ \\ Blanca Delia Arias García ${ }^{3}$ \\ Silvia Susana Robles Montijo ${ }^{4}$
}

Universidad Nacional Autónoma de México, Facultad de Estudios Superiores Iztacala, México

Resumen. Objetivo: describir el significado psicológico de diferentes prácticas de socialización sexual fraterna y determinar si existen diferencias por sexo. Método: estudio transversal con muestreo no probabilístico $(n=$ 214 universitarios); se utilizó la técnica de Redes Semánticas Naturales Modificadas para analizar el significado psicológico de diferentes prácticas de socialización sexual fraterna. Resultados: Las mujeres refieren prácticas y significados centrados en un rol afectivo y los varones en un rol instrumental; sin embargo, existe un amplio espectro de tácticas de socialización que son compartidas por ambos sexos. Conclusiones: el género y la cultura son elementos que deben ser considerados en las prácticas fraternas de socialización sexual.

Palabras clave. Sexualidad, familia, hermanos, relaciones fraternas.

Abstract. Objective: to describe the psychological meaning of diverse of fraternal sexual socialization practices within a group of college students and determine whether there are significant differences among genders. Methods: cross-sectional study, non-probabilistic sample method ( $n=214$ college students) and the Modified Natural Semantic Networks were all used in order to analyze the psychological meaning of different practices of fraternal sexual socialization. Results: female participants were found to refer practices and meanings centered on an affective role, while men were centered on an instrumental role; however, there is a broad spectrum of socialization tactics shared by both men and women. Conclusion: gender and culture play a role in the fraternal sexual socialization practices that should be taken into account.

Keywords. Sexuality, family, gender, siblings, relationship.

Investigación financiada gracias al Programa UNAM-DGAPA-PAPIIT IA301616.

${ }^{1}$ David Javier Enríquez Negrete. Universidad Nacional Autónoma de México, México. Dirección Postal: Av De Los Barrios 1, Los Reyes Ixtacala, Hab Los Reyes Ixtacala Barrio de los Árboles/Barrio de los Héroes, 54090 Tlalnepantla, Méx. Torre Académica de Tutorias, Segundo piso, Cubículo 34. E-mail: david.enriquez@@iztacala.unam.mx

${ }^{2}$ Ricardo Sánchez Medina Clavero. Universidad Nacional Autónoma de México, México. E-mail: ricardo.sanchez@iztacala.unam.mx ${ }^{3}$ Blanca Delia Arias García Universidad Nacional Autónoma de México, México. E-mail: blanca.arias@iztacala.unam.mx

${ }^{4}$ Silvia Susana Robles Montijo. Universidad Nacional Autónoma de México, México. E-mail: susana@unam.mx

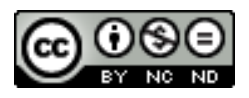

Esta obra está bajo una licencia de Creative Commons Reconocimiento-NoComercial-SinObraDerivada 4.0 Internacional. 


\section{Introducción}

La tasa de fecundidad internacional es de 2.5 hijos por mujer (Organización de las Naciones Unidas, 2014) por tanto una gran proporción de habitantes en el mundo tiene al menos un/a hermano/a. Diariamente los hermanos pasan muchas horas juntos (Tucker, McHale, \& Crouter, 2008) y esto favorece el establecimiento de una de las relaciones más duraderas y significativas en la vida (Craft-Rosenberg, Montgomery, Hill, Kauder, \& Eisbach, 2011). En las familias latinas (cf. Parke \& Buriel, 2007) las relaciones fraternas son centrales porque culturalmente promueve el cuidado y apoyo de los hermanos mayores -principalmente mujeres- hacia los menores (East \& Hamill, 2013), por tanto, en el sistema de relaciones fraternas se desempeñan tareas de cuidado, protección y monitoreo en la ausencia de los padres (Cicirelli, 1994), gracias a que operan a través de valores y comportamientos relacionados con el respeto, el apoyo y asistencia hacia padres y hermanos (Fuligni, 2007).

En estas condiciones, los hermanos menores aprenden de los mayores, valores, conocimientos y habilidades cognitivas, emocionales y sociales para un adecuado ajuste psicosocial (Cicirelli, 1994; Tucker et al., 2008). Así, los hermanos se posicionan como agentes de socialización (McHale, Updegraff, \& Whiteman, 2012) junto con los padres (Grusec \& Davidov, 2010), sin embargo, se ha priorizado el estudio de los mecanismos de socialización parental (cf. Bugental \& Grusec, 2007) sobre los fraternos (Brody, 2004; McHale et al., 2012; Buist, Deković, \& Prinzie, 2013), a pesar de que los hermanos contribuyen en la conformación de la identidad (Davies, 2014), en el aprendizaje de habilidades sociales (Downey, Condron, \& Yucel, 2015), en la auto regulación emocional y en el establecimiento de relaciones interpersonales positivas con otros (Brody, 2004). Aún en la adolescencia y la edad adulta, los efectos de las interacciones entre hermanos pueden observarse sobre la autonomía, la seguridad, la satisfacción con la vida (Hollifield \& Conger, 2014), el establecimiento y calidad de las relaciones románticas (Doughty, McHale, \& Feinberg, 2015) y sobre el comportamiento sexual (Widmer, 1997).

Cuando los agentes de socialización influyen sobre los comportamientos, actitudes, valores, ideas, creencias, símbolos culturales, significados y códigos de conducta relativos a la sexualidad, entonces el fenómeno de estudio se conceptúa como "socialización sexual" (Shtarkshall, Santelli, \& Hirsch, 2007). Este proceso es multidimensional y participan diversos agentes, por ejemplo, padres (Kincaid, Jones, Sterrett, \& McKee, 2012), maestros (Shtarkshall et al., 2007), amigos (Fontenberry, 2013) y medios de comunicación (Ugarte, Högberga, Valladaresc, \& Esséna, 2013). Existe evidencia empírica que documenta que los hermanos también fungen como agentes de socialización sexual (Wallace, Hooper, \& Persad, 2014; Widmer, 1997) y la descripción de las interacciones fraternas y mecanismos de influencia sobre el comportamiento, podrían proporcionar información complementaria sobre el proceso de socialización sexual familiar (McHale et al., 2012).

De acuerdo con Grusec y Davidov (2010), las interacciones de socialización se pueden clasificar por su función evolutiva/adaptativa, en aquellas que tienen como meta la protección, el aprendizaje guiado, el control y la reciprocidad. Por tanto, el presente estudio parte de este enfoque para describir las interacciones de socialización sexual fraternas. 
En la literatura existe evidencia de estas interacciones; por ejemplo, en las relaciones que tienen como propósito la "protección", Wallace, et al. (2014) documentaron cómo los hermanos mayores muestran disposición para asesorar sobre cómo reducir riesgos en las relaciones sexuales, lo cual implicaría una acción dirigida a que sus hermanos menores puedan responder exitosamente a una situación de riesgo. Respecto a las interacciones basadas en el "aprendizaje guiado", Coleman-Minahan y Scandlyn (2015) describieron que los hermanos mayores brindan consejos y comparten sus experiencias sexuales, lo cual probabilizó el uso del condón en sus hermanos menores. Grusec y Davidov (2010) señalan que, cuando el agente de socialización funge como maestro para el aprendizaje de habilidades o internalización de valores hacia otros menos experimentados, se puede ubicar la interacción en este dominio. Las interacciones que tienen como propósito el “control” se basan en la condición de jerarquía (Grusec \& Davidov, 2010). Wallace (2008) reportó que los hermanos mayores suelen presionar a los menores para que se impliquen en actividades sexuales. Finalmente, en las relaciones basadas en la "reciprocidad" se buscan los beneficios sociales y emocionales entre iguales, por ejemplo, afecto positivo (Bugental \& Grusec, 2007), el cual incrementa las probabilidades de que los hermanos hablen entre sí, incluso sobre sexo (Kowal \& Blinn-Pike, 2004) a pesar de lo complicado que puede ser tratar este tema (Wallace, 2008).

Además de considerar las prácticas de socialización sexual fraternas en cada dominio de interacción, se debe tomar en cuenta el tipo de diada -mixta/mismo sexo- dado que los resultados en la socialización son diferenciados. Kowal y Blinn-Pike (2004) describieron que las mujeres perciben actitudes más positivas hacia el sexo cuando reportaban tener una hermana mayor, además es mejor la calidad de la relación y la comunicación sexual, en contraste con las diadas de hombre-hombre y mixtas.

En las diadas de varones, Widmer (1997) señala que, los hermanos menores quienes informaron haber usado métodos anticonceptivos en su primera relación sexual, eran pares de hermanos mayores quienes reportaron protegerse en su debut sexual; por otro lado, la condición de tener hermanos mayores sin experiencia sexual probabilizó que los menores no hubieran debutado sexualmente. Estas diferencias por sexo y diada pueden interpretarse a partir de los estereotipos del rol fraterno (mayor-menor) y de género, los cuales se fomentan, inicialmente, por socialización familiar y posteriormente son perpetuados, parcialmente, por las relaciones entre hermanos (Korneich, Hearn, Rodríguez, \& O’Sullivan, 2003).

Así, las diadas fraternas y los estereotipos de género cobran relevancia en las familias latinas (East \& Hamill, 2013) debido a los valores del familismo que se promueven (Parke \& Buriel, 2007); sin embargo, McHale et al. (2012) evidencian que el estudio de las relaciones fraternas en familias latinas es limitado y existen pocas investigaciones en su contexto cultural. Bugental y Grusec (2007) señalan que la cultura no puede ser negada en el estudio de la socialización, ya que ésta modifica las interacciones entre el agenteobjeto de socialización, por tanto, el papel de los hermanos en este proceso está sujeto a factores relacionados con el género y la cultura. Grusec y Davidov (2010) puntualizan 
que las interacciones de protección, aprendizaje guiado, control y reciprocidad pueden resultar efectivas para la socialización en algunos grupos culturales pero en otros no, esto debido a que cada grupo enfatiza y atribuye diferentes significados a las prácticas y metas de socialización.

De esta forma, las prácticas de socialización sexual fraternas y su significado tendrían una estrecha relación con el contexto cultural, debido a que la cultura delimita comportamientos, pensamientos y emociones de acuerdo con las prescripciones que se articulan a través de los estereotipos (Rocha-Sánchez \& Díaz-Loving, 2005). Así, en el estudio de las relaciones entre hermanos se cruzan dos ejes prescriptivos: los estereotipos de género y del rol fraterno. Por un lado, los estereotipos de género promueven, de forma diferenciada, cómo "deberían de comportarse sexualmente" hombres y mujeres (Caricote, 2006; Ortiz-Torres, Serrano-García, \& Torres-Burgos, 2000) y los estereotipos del rol fraterno (mayor/menor) condicionan las formas de relación entre hermanos; por ejemplo, Enríquez-Negrete, Arias, Sánchez y Robles (2011) analizaron el significado psicológico de "hermano mayor" y "hermano menor" en universitarios; sus resultados muestran que existen cualidades y atributos compartidos que se asocian a ambas figuras, pero existen otros que, son diferentes de acuerdo con los roles instrumental y afectivo que comúnmente ejercen hombres y mujeres en la cultura mexicana (cf. Rocha-Sánchez \& Díaz-Loving, 2005).

La Teoría del Script Social (TSS) propuesta por Abelson (1981) podría explicar cómo a través de guiones culturales internalizados se construyen significados que motivan comportamientos, respuestas y emociones de acuerdo con la situación y la interacción entre hombres, mujeres o ambos. Si bien la TSS se ha utilizado para comprender la conducta sexual de acuerdo con el género (Drury \& Bukowski, 2013) también podría ser utilizada para interpretar las interacciones entre hermanos de acuerdo con el sexo y el rol fraterno, debido a que los scripts socioculturales sirven como modelos cognoscitivos que las personas emplean para guiar y evaluar el comportamiento intra e interpersonal de acuerdo con la interacción y la situación, no importando si se está participando activamente o como observador (cf. Abelson, 1981). En esta línea de pensamiento, hermanos y hermanas podrían ejercer prácticas de socialización sexual, de acuerdo con los scripts sociales de género y del rol fraterno, para socializar sexualmente a sus hermanos/as menores.

Un punto medular es que no importa si los individuos tienen -o no- un hermano/a mayor, de igual forma orientan su comportamiento y cognición a través de scripts cognoscitivos que representan las relaciones fraternas, debido a que éstos son comunicados a través de ejemplos de otros integrantes de la cultura que ya los han internalizado y reproducido - por ejemplo, familiares, amigos, etcétera- o son promovidos a través de los medios de comunicación masiva que transmiten representaciones sobre cómo las personas por ejemplo, hermanos/as- actuarían o responderían ante situaciones particulares, delimitando cualidades de las interacciones, tiempos, lugares, objetivos, secuencias de relación, entre otros elementos que resultarían deseables y aceptados para el grupo cultural (Wiederman, 2005). Así, el papel de la observación y la imitación es fundamental, motivo por el cual, la Teoría del Aprendizaje Social se utiliza frecuentemente como 
marco explicativo para comprender el mecanismo de influencia entre hermanos para la socialización sexual fraterna, según la revisión realizada por Enríquez-Negrete y Sánchez (2015). Así, para avanzar en la descripción de aquellas prácticas de socialización sexual que se asocian al ejercicio del rol fraterno en diferentes dominios de interacción -protección, aprendizaje guiado, control, y reciprocidad- y conocer el sentido y significado que hombres y mujeres atribuyen a dichas acciones en el seno de la cultura latina, es preciso desarrollar investigaciones que permitan valorar el papel que los hermanos tienen como agentes de socialización sexual familiar.

Objetivo

Describir las prácticas de socialización sexual fraternas asociadas a los dominios de interacción propuestos por Grusec y Davidov (2010) y determinar por sexo, el significado psicológico que estudiantes de nivel superior atribuyen a estas prácticas.

\section{Método}

\section{Participantes}

Participaron 214 universitarios (37.9\% hombres y 62.1\% mujeres) adscritos a dos universidades públicas de la Ciudad de México; tenian en promedio 19.91 años ( $D E=$ 1.68, Rango $=18-24)$, en su mayoría solteros $(97.2 \%)$ y provenientes de familias biparentales (65\%). El 47.7\% informó tener al menos un hermano, el 37.9\% dos y $14.4 \%$ entre tres y siete. El 36.2\% reportó tener solamente hermanos mayores, $44.1 \%$ únicamente hermanos menores y $19.7 \%$ informaron tener tanto hermanos mayores como menores.

Selección y tamaño de la muestra

Muestreo no probabilístico intencionado y por conveniencia (Banerjeey \& Chaudhury, 2010) dado que los directivos de las universidades eligieron los grupos de alumnos de acuerdo con la disponibilidad. Se utilizó el software GPower v.3.1 para estimar el tamaño de la muestra (cf. Faul, Erdfelder, Lang, \& Buchner, 2007) a partir de los parámetros mínimos aceptados (Kadamy \& Bhalerao, 2010). El tamaño del efecto de $\chi^{2}$ fue estimado para un efecto grande $(w=.50)$ de acuerdo con lo estipulado por Cárdenas \& Arancibia (2014). El valor de $\alpha$ se calculó en .01 (Kadamy \& Bhalerao, 2010), y el poder de la prueba o potencia estadística $(1-\beta)$ se estimó por arriba del valor mínimo aceptado (.80) calculándose en .95 (Faul et al., 2007; Faul, Erdfelder, Lang, \& Buchner, 2009) con 1 grado de libertad (k-1). Así, con un valor de parámetro de no centralidad de $\lambda=18$, un valor crítico de $\chi^{2}=6.63$, el tamaño de la muestra $(n)$ requerido para contrastar los grupos, con probabilidad de 1\% de cometer Error Tipo I, es de 72 universitarios. Sin embargo, se buscó incrementar el tamaño de la muestra para evitar la perdida de datos cuando no se cumplieran los criterios de inclusión a la muestra.

Criterios de inclusión y exclusión de la muestra

Inclusión: a) tener entre 18-24 años; b) estar inscrito en el plantel educativo donde se llevó a cabo la evaluación; y c) tener al menos un hermano/a. Exclusión: a) no firmar el consentimiento informado; b) no contestar completamente el cuadernillo de evaluación; y c) reportar algún impedimento para participar en el estudio. 
Tipo de estudio

El estudio fue de tipo transversal-descriptivo-comparativo-observacional (Méndez, Namihira, Moreno, \& Sosa, 2001).

\section{Instrumentos}

Se utilizó la técnica de las Redes Semánticas Naturales Modificadas (Reyes-Lagunes, 1993) para conocer el significado psicológico de diferentes estímulos asociados con prácticas fraternas de socialización sexual y para identificar muestras de conductas que una población meta considera perteneciente a un constructo de interés. La técnica consiste en brindar una palabra/frase central -o estímulo- y a partir de éste se genera una lista de palabras relacionadas con el estímulo presentado (Figueroa, González, \& Solís, 1981). Posteriormente se solicita al participante que jerarquice las definidoras de acuerdo con el orden de importancia en relación al estímulo presentado, lo anterior permite estimar la relevancia de las palabras utilizadas para definir la red semántica (Peso Semántico) y así se obtiene un mapeo de cómo los individuos organizan la información. Posteriormente se estima la Distancia Semántica Cuantitativa (DSC) para comparar la relevancia de las palabras al interior de la red semántica. Los estímulos presentados fueron "Lo que hace un hermano mayor para...": a) enseñar sexualidad; b) restringir la vida sexual del hermano menor; c) mostrar interés en la vida sexual del hermano menor; y d) proteger de los peligros del sexo al hermano menor.

\section{Procedimiento}

La Comisión de Ética de la Facultad de Estudios Superiores Iztacala de la Universidad Nacional Autónoma de México (UNAM) aprobó y autorizó previamente el desarrollo del estudio. Se asistió a las universidades para realizar la evaluación colectiva en cada uno de los grupos asignados por la dirección de las instituciones educativas. Se solicitó la firma del consentimiento informado en el cuadernillo de evaluación y se hizo énfasis en la participación voluntaria y anónima; destacando que los datos serían utilizados para fines de investigación. El tiempo promedio para contestar el cuadernillo fue de 25 minutos.

\section{Análisis de datos}

Primero se ordenaron alfabéticamente las palabras, posteriormente se contabilizaron para conocer el Tamaño de Red (TR) por estímulo. A partir de la frecuencia de las palabras utilizadas y de la jerarquización de éstas, se calculó el Peso Semántico (PS) para determinar la importancia de las definidoras en la red semántica. Se utilizó el punto de quiebre propuesto por Cattell para el análisis factorial con el propósito de seleccionar aquellas palabras que constituirían cada red. Con estos vocablos se estimó la DSC para cada definidora asignándole el 100\% al PS más alto y por regla de tres se estimaron los valores subsecuentes del resto de las definidoras. A continuación se restó 100 como una constante, con el propósito de que los valores cercanos a cero se interpretaran como palabras con mayor relevancia en la red. Utilizando la Ji Cuadrada se comparó por sexo el TR entre hombres y mujeres y se estimó la rs de Spearman para determinar si los PS de las definidoras que se repiten en cada sexo, se asocian con el orden asignado para definir el estímulo.. De acuerdo con Reyes-Lagunes (1993), las definidoras obtenidas en la 
conformación de cada red, se pueden clasificar como positivas, negativas y descriptivas; y/o como definidoras pertenecientes a la dimensión conductual, afectiva o cognitiva, tomando como punto de referencia el estímulo.

\section{Resultados}

Ante el estímulo "Lo que hace un hermano mayor para enseñar sexualidad", hombres $(\mathrm{TR}=169)$ y mujeres $(\mathrm{TR}=176)$ utilizaron sólo definidoras positivas (Figura 1$)$, no variando significativamente el TR por sexo $\left(\chi^{2}=.142, p>.05\right)$. Las palabras incluidas en la red semántica por varones y mujeres, en su mayoría fueron propias de la dimensión conductual y sólo definidoras como "información", "confianza" y "responsabilidad" son de naturaleza cognitiva. Para hacer una distinción por sexo en el tipo de palabras utilizadas para definir el estímulo, en la Figura 1 se subrayan las definidoras que son diferentes para hombres y mujeres, y aquellas que se encuentran sin subrayar son compartidas por ambos sexos.

El coeficiente de correlación de Spearman mostró que no existe una correlación estadísticamente significativa entre los PS de las definidoras que se repiten por sexo $(\mathrm{rs}=.450, p>.05)$, es decir, a pesar de que utilizan las mismas palabras para definir la red semántica, la importancia que atribuyen a éstas es diferente.

Respecto al segundo estímulo "Lo que hace un hermano mayor para restringir la vida sexual del hermano menor" se encontraron diferencias estadísticamente significativas en el tamaño de red entre hombres $(T R=171)$ y mujeres $(T R=224)$, siendo mayor el número
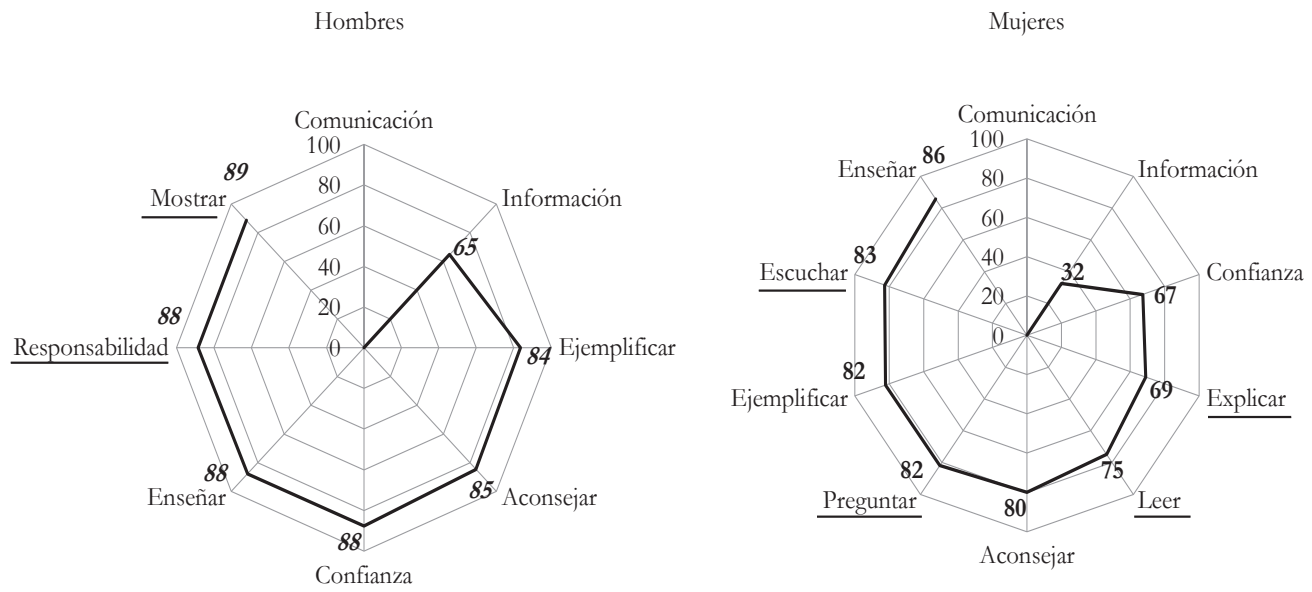

Figura 1. Definidoras y Distancia Semántica Cuantitativa de hombres y mujeres en el estímulo perteneciente al dominio de aprendizaje guiado. 
de palabras utilizadas por las universitarias $\left(\chi^{2}=7.111, p<.05\right)$. Los varones, en su mayoria utilizaron definidoras negativas (ocho palabras) y solo utilizaron "comunicación", "protección”, "información” y "cuidado" como definidoras positivas.

Por su parte, las mujeres utilizaron una cantidad similar de definidoras positivas (cinco) y negativas (seis). Entre las positivas se encuentran "comunicación", "información", "advertir", "cuidar" y "protección" (Figura 2). En la Figura 2 se hace la distinción de aquellas palabras que usan hombres mujeres para definir el estímulo, las cuales se encuentran subrayadas en cada una de las redes. En tanto, las definidoras que no se encuentran subrayadas son aquellas que son compartidas por ambos sexos para definir la frase.
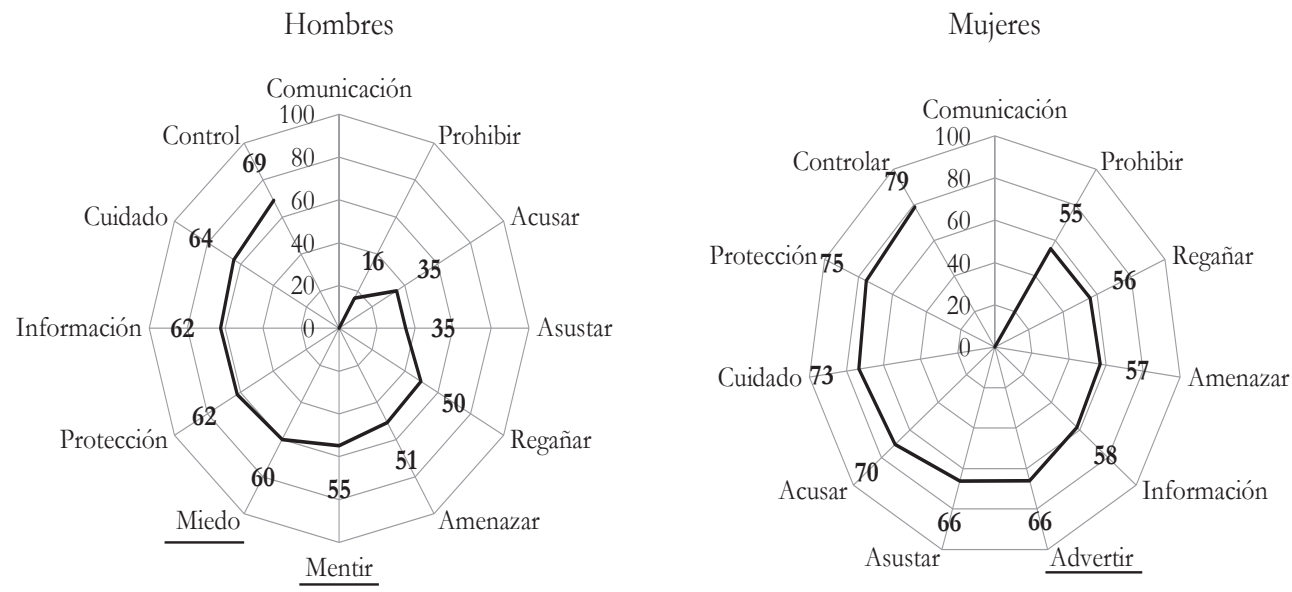

Figura 2. Definidoras y Distancia Semántica Cuantitativa de hombres y mujeres en el estímulo perteneciente al dominio de control.

Ambos sexos refirieron las definidoras como conductuales, sólo "información" se ubico como un vocablo de naturaleza cognoscitiva y utilizaron "miedo" como una palabras de orden emocional. La prueba estadística de correlación $(r s=.789, p<.05)$ indica que existe una asociación significativa entre los valores del PS en hombres y mujeres para este estímulo. Es decir, el PS que se atribuye a las definidoras compartidas son similares sin importar el sexo.

En el estímulo "Lo que hace un hermano mayor para mostrar interés en la vida sexual del hermano menor" no se encontraron diferencias estadísticamente significativas en el tamaño de red por sexo (TR hombres $=154$, TR mujeres $=181, \chi^{2}=7.111, p>.05$ ). Ambos sexos utilizaron definidoras positivas y cognoscitivas como "información” y "confianza", el resto de las palabras utilizadas fueron relacionadas con comportamientos (Figura 3). Asimismo, existe una asociación estadísticamente significativa entre los PS de ambas redes semánticas ( $\mathrm{rs}=.776, p<.05)$, lo cual implica que la importancia que hombres y mujeres atribuyen a las definidoras que comparten es similar. 
Hombres

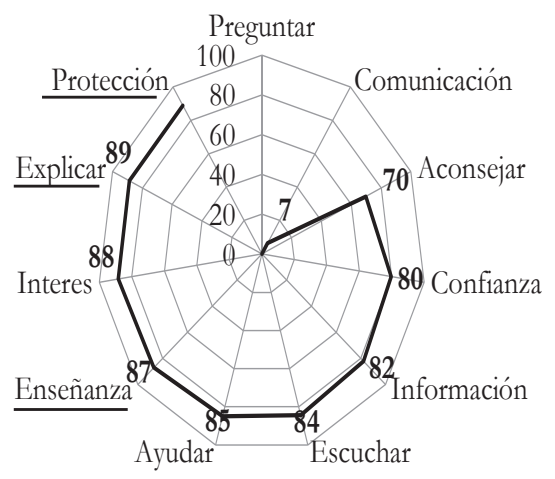

Mujeres

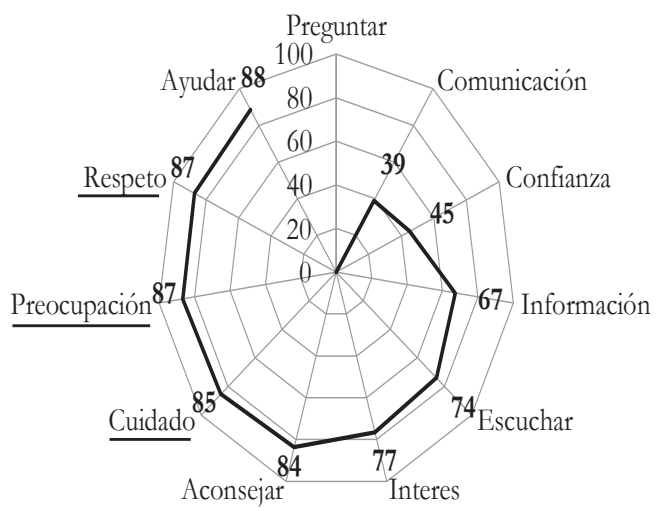

Figura 3. Definidoras y Distancia Semántica Cuantitativa de hombres y mujeres en el estímulo perteneciente al dominio de reciprocidad.

En la Figura 3 se subrayan las definidoras que son diferentes para hombres y mujeres, las palabras que se encuentran sin subrayar son los vocablos compartidos por sexo . Finalmente, en el estímulo "Lo que hace un hermano mayor para proteger de los peligros del sexo al hermano menor" el TR para los varones fue de 149 definidoras y en mujeres 179 , no se encontraron diferencias significativas por sexo con respecto al TR $\left(\chi^{2}=2.743\right.$, $p>$.05). Los hombres utilizaron mayoritariamente definidoras positivas (doce) aunque aparecen palabras como "prohibición", "ITS" y "asustar" que se constituyen como palabras negativas. La mayor parte de las palabras son referidas a "acciones" y en menor medida vocablos asociados a aspectos cognoscitivos, como por ejemplo "información” y "confianza"; finalmente "asustar" se ubica como una definidora de orden emocional. De forma similar, las mujeres refieren palabras con carga positiva en la mayor parte de la red semántica y sólo aparece "ITS" con carga negativa. Cabe destacar que en ambos sexos aparecen palabras descriptivas como "anticonceptivos" e "ITS" (Figura 4).

No se encontró correlación significativa entre el PS de las definidoras compartidas entre hombres y mujeres ( $\mathrm{rs}=.545, p>.05$ ), lo cual indica que la relevancia de las palabras compartidas en ambos sexos es diferente. Para hacer una distinción por sexo en el tipo de palabras utilizadas para definir el estímulo, en la Figura 4 se subrayan las definidoras que son diferentes para hombres y mujeres, y aquellas que se encuentran sin subrayar son compartidas por ambos sexos. 
Hombres

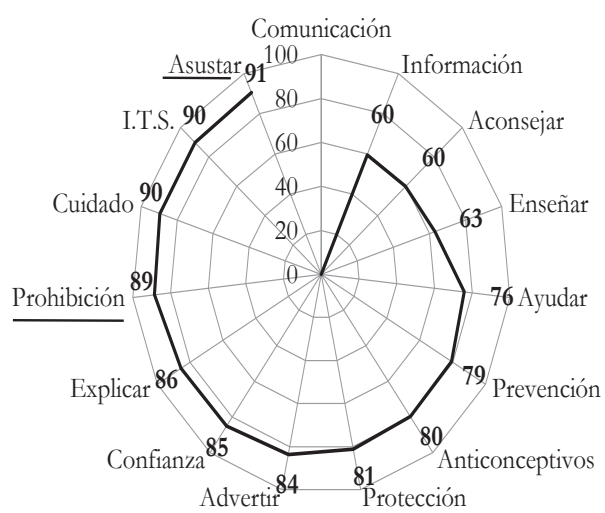

Mujeres

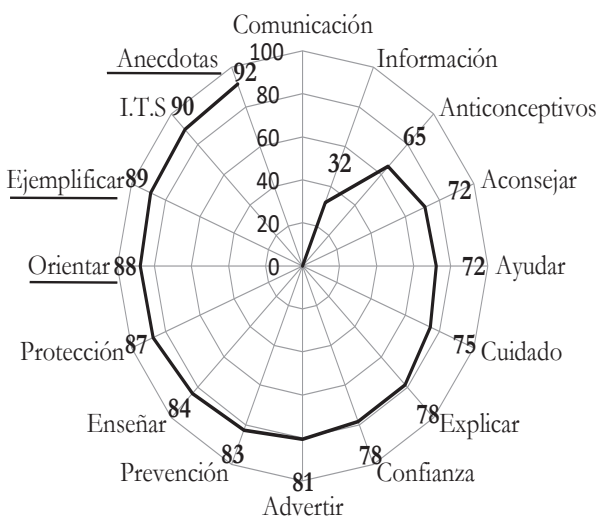

Figura 4. Definidoras y Distancia Semántica Cuantitativa de hombres y mijeres en el estímulo perteneciente al dominio de protección.

\section{Discusión}

Para fines expositivos está sección se discute en tres apartados organizados de acuerdo con los resultados obtenidos.

\section{Comunicación e información para la socialización sexual fraterna}

Los participantes sin importar el sexo y el dominio de socialización -protección, aprendizaje guiado, control y reciprocidad- definieron todos los estímulos con las palabras "comunicación" e "información", utilizando estos vocablos en los primeros lugares para definir todas las redes semánticas, lo cual podría estar asociado con la relevancia que tienen estas definidoras para representar las interacciones fraternas; al mismo tiempo que brindan información, de acuerdo con Reyes-Lagunes (1993) sobre manifestaciones cognoscitivas y conductuales que definen un constructo, en este caso particular, las prácticas de socialización sexual del hermano mayor.

Es probable que la "comunicación" se asocie como definidora en todas las redes semánticas dado que es un atributo característico de las relaciones fraternas; Spitze y Trent (2016) demostraron que, en diferentes transiciones familiares, la comunicación entre hermanos se mantiene como un rasgo fraterno relativamente estable a lo largo de la vida. Así, la palabra "comunicación” podría representar este atributo característico, pero al mismo tiempo denotar una práctica común en la socialización sexual entre hermanos. Sin importar el dominio de interacción para la socialización sexual -protección, aprendizaje guiado, control y reciprocidad- y el sexo, la comunicación fue un referente compartido. 
Fowler (2009) describió que la comunicación entre hermanos jóvenes y adultos se encuentra motivada por una necesidad afectiva de demostrar aprecio e interés hacia el otro. Así, la comunicación es un atributo que se encuentra relacionado con el nivel de afectividad entre hermanos (Kowal \& Blinn-Pike, 2004), por tanto, aprovechando la condición emocional fraterna, es probable que los hermanos mayores generen actos comunicativos para proteger a sus hermanos/as de los riesgos potenciales del sexo -dominio de protección-; como un medio para interesarse en su vida íntima -dominio de reciprocidad-; como un recurso para enseñar habilidades/conocimientos relacionados con el acto sexual -dominio de aprendizaje guiado-; o incluso como un medio para restringir algunos comportamientos de riesgo para la salud sexual de los hermanos menores - control-. Sin embargo, es necesario realizar investigación empírica para determinar la intencionalidad y las metas que tienen los hermanos mayores cuando generan actos comunicativos con los hermanos menores, con el propósito de validar si la comunicación podría identificarse como una práctica de socialización sexual genérica en los dominios de socialización sexual (cf. Grusec \& Davidov, 2010).

Cabe destacar, que la comunicación, como una práctica de socialización sexual, podría estar influenciada por las demandas culturales que prescriben que los hermanos mayores se comporten como maestros, mentores y amigos (Craft-Rosenberg et al., 2011; EnríquezNegrete et al., 2011), y en consecuencia, la comunicación sería un atributo característicos del ejercicio de estos roles. Dada la relevancia y la frecuencia de aparición de esta definidora en todas las redes semánticas, se sugiere valorar el papel que juegan los hermanos en contraste con otros agentes de socialización y en relación a la comunicación. Widman, Choukas-Bradley, Helms, Golin, \& Prinstein (2014) demostraron que la frecuencia de las discusiones sobre sexo con los amigos, los padres y la pareja es diferenciada. En el estudio de Widman et al. (2014) no se toma en cuenta a los hermanos, a pesar de que éstos con frecuencia discuten sobre sexo (Kowal \& Blinn-Pike, 2004; Wallace, 2008) incluso con mayor comodidad que cuando hablan con sus padres sobre los mismos temas (Wallace, 2008). Por tanto, desarrollar investigación que permita describir con precisión en qué condiciones y circunstancias la comunicación sexual fraterna tiene relación con las creencias (Kowal \& Blinn-Pike, 2004) y comportamientos relacionados con la prevención de diferentes problemas de salud sexual (Coleman-Minahan \& Scandlyn, 2015) podría constituirse una vía para diferenciar el papel de la comunicación sexual fraterna en contraste con las discusiones que se tienen con los padres, amigos y la pareja, lo cual abre un campo de investigación fértil dado que los estudios que versan en torno a los motivos y actos comunicativos de los hermanos es limitada (Fowler, 2009).

Otra definidora común en todas las redes semánticas, independientemente del sexo y del dominio de socialización, fue la "información", la cual tiene una estrecha relación con la "comunicación". Ybarra, Emenyonu, Nansera, Kiwanuka y Bangsberg (2008) puntualizan que los hermanos mayores son una de las principales fuentes de información sobre aspectos relacionados con la salud sexual y la prevención del VIH/SIDA, ya que éstos se encuentran en una posición privilegiada para compartir experiencias y opiniones similares en torno al sexo -siempre y cuando no haya mucha diferencia en edades- (Kowal \& Blinn-Pike, 2004). La información que se pueda obtener de un hermano (o de cualquier otra fuente) es relevante en la medida en que se convierte en una condición necesaria 
para saber qué hay que hacer para estar saludables y evitar riesgos (Fisher \& Fisher, 1992), siempre y cuando ésta sea veraz y oportuna. El reto que surge a continuación es investigar como la información se relaciona con la construcción y predicción de variables motivacionales y conductuales en la socialización y alfabetización sexual fraterna. Los resultados de este estudio solo evidencian que la "información" es un referente asociado a diferentes prácticas de socialización sexual fraterna, pero no aportan datos para construir afirmaciones relacionales o causales sobre la comunicación y sus efectos en la conducta sexual de los hermanos.

\section{La especificidad de dominio en la socialización sexual fraterna}

Se puede observar la repetición de diversas definidoras en todas las redes semánticas, es decir, hombres y mujeres usan referentes similares -por ejemplo, comunicación, información, aconsejar, confianza y enseñar- para definir los estímulos, los cuales representan acciones en diferentes dominios de socialización sexual, de acuerdo con el enfoque propuesto por Grusec y Davidov (2010). Para valorar si cada uno de los referentes conductuales -prácticas de socialización- obtenidos en las redes semánticas, tienen o no, relación con los dominios específicos de socialización propuestos Grusec y Davidov (2010) es necesario, además de identificar la práctica, evaluar la meta y el resultado de la interacción para la socialización.

Ejemplificando, para comprender si la "comunicación" pertenece a un dominio de protección, reciprocidad, aprendizaje guiado o control se debe de conocer el propósito del acto comunicativo, no es lo mismo hablar con el hermano menor para enseñarlo a usar un condón (dominio de aprendizaje guiado) que para persuadirlo e inhibir sus deseos de tener múltiples parejas (dominio de control). Si la práctica de socialización es congruente con la meta que se pretende alcanzar, solo restaría evaluar el resultado, es decir, si la comunicación sexual cumple con el propósito de enseñar a usar un condón (p. ej. aprendizaje guiado) el resultado esperado sería que los hermanos menores utilizaran preservativo en el acto sexual. Así, se torna imperante trascender la identificación de la práctica de socialización sexual fraterna y valorar su ocurrencia, frecuencia, la intencionalidad del acto y el resultado. De acuerdo con lo anterior, es necesario realizar estudios donde se incluyan ambos agentes de la interacción fraterna y se valore por un lado las prácticas de socialización sexual del hermano mayor y su intencionalidad, y por otro, los resultados obtenidos en el comportamiento sexual del hermano menor; lo anterior con el propósito de buscar la congruencia entre dominios de socialización-prácticas fraternas-resultados. Solo así, se avanzará en la comprensión de la socialización sexual desde este enfoque, sin pasar por alto el sentido y significado que el contexto cultural atribuye a las prácticas y metas de socialización.

\section{Diferencias de género en el significado y las prácticas de socialización sexual fraternas}

Otro factor que debe de ser considerando en el análisis de las prácticas de socialización sexual fraterna es el rol de género (Korneich et al., 2003). Las pruebas de correlación indican que hombres y mujeres atribuyen diferente nivel de importancia a palabras que ambos utilizaron para definir los estímulos "Lo que hace un hermano mayor para proteger de los peligros del sexo al hermano menor" y "Lo que hace un hermano mayor para enseñar sexualidad". Las mujeres obtuvieron puntajes más altos en la DSC en palabras que estuvieron asociadas con atributos y comportamientos que se esperan de ellas a nivel 
cultural, tales como "comunicación”, "información”, "confianza”, "aconsejar", "ayudar”, "cuidado" y "explicar"; esto podría explicarse dado que en la cultura mexicana se fomenta en las mujeres un rol afectivo, basado en las actividades relativas al cuidado de los otros (Rocha-Sánchez \& Díaz-Loving, 2005) lo cual se ve reforzado por los valores de atención y apoyo que promueve el familismo (Parke \& Buriel, 2007).

Estos resultados podrían estar relacionados con el "comportamiento de custodia familiar" (Hagestad, 1986 en Fowler, 2009) en el cual, por factores culturales y de género, las mujeres asumen la responsabilidad de garantizar la cercanía continua entre los miembros de la familia. Así, las prácticas de socialización sexual fraternas por parte de ellas, podrían estar motivadas por componentes culturales, relacionales y emocionales.

En contraste, Floyd (1996) sugiere que los varones se expresan y relacionan de forma diferente, ellos buscan compartir intereses y actividades para demostrar cercanía y afecto con sus hermanos. Considerando el rol instrumental que impronta la cultura a los hombres, éstos son educados para ser autónomos, orientados al logro, fuertes, exitosos y proveedores (Rocha-Sánchez \& Díaz-Loving, 2005). Estas condiciones podrían brindar una interpretación para comprender porque los varones utilizan definidoras centradas en el "hacer" y la "acción" como por ejemplo, "enseñar", "proteger" y "ejemplificar", como parte de su rol instrumental. Y definidoras como "miedo" y "mentir" para obtener control psicológico sobre sus hermanos, aspecto que podría referenciar la supremacía y el poder sociocultural que tiene el varón en la cultura mexicana (Diaz-Guerrero, 1994).

Estas condiciones podrían favorecer que los varones perpetúen los roles tradicionales de género en la socialización sexual con las hermanas menores. Korneich et al. (2003) documentaron que cuando se tienen hermanos mayores y hermanas menores, el comportamiento de ellas se torna auto restrictivo y existe una falta de interés por aspectos relacionados con el sexo, al mismo tiempo que ellos se tornan protectores y vigilan a sus hermanas para evitar que se relacionen con parejas. Incluso, en el estímulo "Lo que hace un hermano mayor para mostrar interés en la vida sexual del hermano menor" los varones utilizaron la definidora "protección" en comparación con la red semántica de las mujeres donde dicho vocablo no apareció.

En resumen, diversas tácticas de socialización sexual y el sentido de éstas, son compartidos por mujeres y varones, aunque las definidoras tienen mayor relevancia para las mujeres, probablemente por la prescripción de género y por socialización familiar. Sin embargo, también existen significados y prácticas fraternas propias de cada sexo, lo cual referencia los roles tradicionales de género. Es importante considerar que el análisis realizado en este estudio brinda información parcial del fenómeno diádico fraterno, dado que solamente se evaluó el significado psicológico y muestras de manifestaciones conductuales relacionadas con diversas prácticas de socialización sexual fraterna en los diferentes dominios propuestos por Grusec y Davidov (2010); sin embargo es indispensable valorar si las prácticas y el significado psicológico son diferentes en edades más tempranas (cf. Fowler, 2009), si éstas varían por la diferencia de edades entre hermanos y por factores asociados al sistema familiar (cf. McHale et al., 2012) o por el tipo de diada -diferente o mismo sexo- (Floyd, 2009; Korneich et al., 2003; Kowal \& Blinn-Pike, 2004; McHale et al., 2012, Widmer, 1997). 


\section{Consideraciones finales}

Si bien en este estudio se utilizó una frase para representar cada uno de los dominios de socialización propuestos por Grusec y Davidov (2010), el estímulo presentado resulta incipiente y no representativo de la complejidad de cada uno de los dominios; sin embargo, este estudio se torna una primera aproximación al estudio de la socialización sexual fraterna desde este enfoque, por tanto, se sugiere profundizar y valorar el espectro de respuestas que puedan derivarse ante estímulos diferentes asociados con los mismos dominios. Cabe destacar que Grusec y Davidov (2010) proponen un quinto dominio participación grupal-, sin embargo, no se consideró la inclusión de un estímulo para evaluar esta dimensión dado que Reyes-Lagunes (1993) sugiere que en las RSNM se presenten máximo cuatro estímulos y un distractor, en consecuencia, se omitió esta dimensión. Se sugiere evaluar el significado y las prácticas de socialización sexual fraternas asociadas a la "participación grupal" y contrastarlas con el espectro de comportamientos propios de los otros dominios.

\section{Referencias}

Abelson, R. P. (1981). Psychological status of the script concept. American Psychologist, 36(7), 715-729. doi:10.1037/0003-066X.36.7.715

Banerjeey, A., \& Chaudhury, S. (2010). Statistics without tears: Populations and samples. Industrial Psychiatry Journal, 19(1), 60-65. doi: 10.4103/09726748.77642

Brody, G. H. (2004). Siblings' Direct and Indirect Contributions to Child Development. Current Directions in Psychological Science, 13(3), 124-126. doi:10.1111/j.0963-7214.2004.00289.x

Bugental, D. B., \& Grusec, J. E. (2007). Socialization Processes. En N. Eisenberg (Ed.), Handbook of Child Psychology. Volume III. Social Emotional, and Personality Development (pp. 366-428). doi:10.1002/9780470147658.chpsy0307

Buist, K. L., Deković, M., \& Prinzie, P. (2013). Sibling relationship quality and psychopathology of children and adolescents: A meta-analysis. Clinical Psychology Review, 33(1), 97-106. doi:10.1016/j.cpr.2012.10.007

Cárdenas, M. \& Arancibia, M. (2014). Potencia estadística y cálculo del tamaño del efecto en $G^{*}$ Power: Complementos a las pruebas de significación estadística y su aplicación en psicología. Salud \& Sociedad, 5(2), 210-224.

Caricote, E. (2006). Influencia de los estereotipos de género en la salud sexual en la adolescencia. Educere, 34(10), 463-470.

Cicirelli, V. G. (1994). Sibling Relationships in Cross-Cultural Perspective. Journal of Marriage and Family, 56(1), 7-20. doi:10.2307/352697

Coleman-Minahan, K., \& Scandlyn, J. (2015). The role of older siblings in the sexual and reproductive health of Mexican-origin young women. Contraception, 92(4), 392-393. doi:10.1016/j.contraception.2015.06.154 
Craft-Rosenberg, M., Montgomery, L. A., Hill, J., Kauder, J., \& Eisbach, S. (2011). Sibling Death/Loss. En M. Craft-Rosenberg \& S. Pehler (Eds.), Encyclopedia of Family Health (pp. 951-957). doi:10.4135/9781412994071.n311

Davies, K. (2014). Siblings, Stories and the Self: The Sociological Significance of Young People's Sibling Relationships. Sociology, 49(4), 679-695. doi:10.1177/0038038514551091

Díaz-Guerrero, R. (1994). Psicología del mexicano. Descubrimiento de la etnopsicología. México: Trillas.

Doughty, S. E., McHale, S. M., \& Feinberg, M. E. (2015). Sibling Experiences as Predictors of Romantic Relationship Qualities in Adolescence. Journal of Family Issues, 36(5), 589-608. doi:10.1177/0192513X13495397

Downey, D. B., Condron, D. J., \& Yucel, D. (2015). Number of Siblings and Social Skills Revisited Among American Fifth Graders. Journal of Family Issues, 36(2), 273-269. doi:10.1177/0192513X13507569

Drury, K. M., \& Bukowski, W. M. (2013). Sexual Development. En D. S. Bromberg \& W. T. O'Donohue (Eds.) Handbook of Child and Adolescent Sexuality (pp.115-144). doi:10.1016/B978-0-12-387759-8.00005-2

East, P. L., \& Hamill, S. B. (2013). Sibling Caretaking Among Mexican American Youth: Conditions That Promote and Hinder Personal and School Success. Hispanic Journal of Behavioral Sciences, 35(4), 542-564. doi:10.1177/0739986313499003

Enríquez-Negrete, D., \& Sánchez, R. (2015). Influencia de los hermanos en el comportamiento sexual de riesgo y preventivo: una revisión. Revista Digital Internacional de Psicología y Ciencia Social, 2(1), 113-132. doi: 10.22402/j.rdipycs. unam.2.1.2016.59.113-125

Enríquez-Negrete, D., Arias, B., Sánchez, R., \& Robles, S. (junio, 2011). Significado de las relaciones fraternas: rol de los hermanos mayores y menores. Memoria en extenso, XXXIII Congreso Interamericano de Psicología, Medellín, Colombia.

Faul, F., Erdfelder, E., Lang, A., \& Buchner, A. (2007). G*Power 3:A flexible statistical power analysis program for the social, behavioral, and biomedical sciences. Behavior Research Methods, 39(2), 175-191. doi:10.3758/BF03193146

Faul, F., Erdfelder, E., Lang, A., \& Buchner, A. (2009). Statistical power analyses using $G^{*}$ Power 3.1: Tests for correlation and regression analyses. Behavior Research Methods, 41(4), 1149-1160. doi:10.3758/BRM.41.4.1149

Figueroa, J., González, E., \& Solís, V. (1981). Una aproximación al problema del significado: las redes semánticas. Revista latinoamericana de psicología, 13(3), 447-458.

Fisher, J. D., \& Fisher, W. A. (1992). Changing AIDS-risk behavior. Psychological Bulletin, 111(3), 455-474. doi:10.1037/0033-2909.111.3.455 
Floyd, K. (2009). Communicating closeness among siblings: An application of the gendered closeness perspective. Journal Communication Research Reports, 13(1), 27-34. doi:10.1080/08824099609362067

Fontenberry, J. D. (2013). Sexual Development in Adolescents. En D. S. Bromberg \& W. T. O'Donohue (Eds.), Handbook of Child and Adolescent Sexuality (pp. 171-192). doi:10.1016/B978-0-12-387759-8.00007-6

Fowler, C. (2009). Motives for Sibling Communication Across the Lifespan. Communication Quarterly, 57(1), 51-66. doi: 10.1080/01463370802662499

Fuligni, A. J. (2007). Family Obligation, College Enrollment, and Emerging Adulthood in Asian and Latin American Families. Child Development Perspectives, 1(2), 96-100. doi:10.1111/j.1750-8606.2007.00022.x

Grusec, J. E., \& Davidov, M. (2010). Integrating Different Perspectives on Socialization Theory and Research: A Domain-Specific Approach. Child Development, 81(3), 687-709. doi:10.1111/j.1467-8624.2010.01426.x

Hollifield, C. R., \& Conger, K. J. (2014). The Role of Siblings and Psychological Needs in Predicting Life Satisfaction During Emerging Adulthood. Emerging Adulthood, 3(3), 143-153. doi:10.1177/2167696814561544

Kadamy, P., \& Bhalerao, S. (2010). Sample size calculation. International Journal for Ayurveda Research, 1(1), 55-57. doi: 10.4103/0974-7788.59946

Kincaid, C., Jones, D. J., Sterrett, E., \& McKee, L. (2012). A review of parenting and adolescent sexual behavior: The moderating role of gender. Clinical Psychology Review, 32(3), 177-188. doi:10.1016/j.cpr.2012.01.002

Korneich, J. L., Hearn, K. D., Rodriguez, G., \& O’Sullivan, L. F. (2003). Sibling influence, gender roles, and the sexual socialization of urban early adolescent girls. The Journal of Sex Research, Gender and Sexuality, 40(1), 101110. doi:10.1080/00224490309552170

Kowal, A. K., \& Blinn-Pike, L. (2004). Sibling Influences on adolescents' attitudes toward safe sex practices. Family Relations, 53(4), 377-384. doi:10.1111/ j.0197-6664.2004.00044.x

McHale, S. M., Updegraff, K. A., \& Whiteman, S. D. (2012). Sibling Relationships and Influences in Childhood and Adolescence. Journal of Marriage and Family, 74(5), 913-930. doi:10.1111/j.1741-3737.2012.01011.x

Méndez, I., Namihira, D., Moreno, L., \& Sosa, C. (2008). Elprotocolo de investigación. Lineamientos para su elaboración y análisis. México: Trillas.

Organización de las Naciones Unidas (ONU, 2014). La situación demográfica en el mundo, 2014. (Informe conciso ST/ESA/SER.A/354). Recuperado de http:// www.un.org/en/development/desa/population/publications/pdf/ trends/Concise $\% 20$ Report $\% 20$ on $\% 20$ the $\% 20$ World $\% 20$ Population $\% 20$ Situation \%202014/es.pdf 
Ortiz-Torres, B., Serrano-Garcia, I., \& Torres-Burgos, N. (2000). Subverting culture: Promoting HIV/AIDS prevention among Puerto Rican and Dominican women. American Journal of Community Psychology, 28(6), 859-881. doi:10.1023/A:1005167917916

Parke, R. D., \& Buriel, R. (2007). Socialization in the Family: Ethnic and Ecological Perspectives. En N. Eisenberg (Ed.), Handbook of Child Psychology. Volume III. Social Emotional, and Personality Development (pp. 429-504). doi:10.1002/9780470147658.chpsy0308

Reyes-Lagunes, I. (1993). Redes semánticas para la construcción de instrumentos. Revista de Psicología Social y Personalidad, 9(1), 83-99.

Rocha-Sánchez, T., \& Díaz-Loving, R. (2005). Cultura de género: La brecha ideológica entre hombres y mujeres. Anales de Psicología, 21 (1), 42-49.

Shtarkshall, R. A., Santelli, J. S., \& Hirsch, J. S. (2007). Sex education and sexual socialization: roles for educators and parents. Perspectives on Sexual and Reproductive Health, 39(2), 116-119. doi:10.1363 / 3911607

Spitze, G. D., \& Trent, K. (2016). Changes in Individual Sibling Relationships in Response to Life Events. Journal of Family Issues, 39(2), 1-24. doi:10.1177/0192513X16653431

Tucker, C. K., McHale, S. M., \& Crouter, A. C. (2008). Links between older and younger adolescent siblings' adjustment: The moderating role of shared activities. International Journal of Behavioral Development, 32(2), 152-160. doi:10.1177/0165025407087214

Ugarte, W. J., Högberga, U. Valladaresc, E., \& Esséna, B. (2013). Assessing knowledge, attitudes, and behaviors related to HIV and AIDS in Nicaragua: A community-level perspective. Sexual \& Reproductive Healthcare, 4(1), 37-44. doi:10.1016/j.srhc.2012.11.001

Vargas-Garduño, M.; Méndez Puga, A.; Vargas Silva, A. (2014). La técnica de las redes semánticas naturales modificadas y su utilidad en la investigación cualitativa. Memorias en extenso, IV Encuentro Latinoamericano de Metodología de las Ciencias Sociales, Costa Rica. Recuperado de http:// www.memoria.fahce.unlp.edu.ar/trab_eventos/ev.8204/ev.8204.pd

Wallace, S. A. (2008). 63: I Am My Brother's Keeper: Sibling Influences on Sexual Attitudes and Behaviors among Urban Black Youth. Journal of Adolescent Health, 42(2), 43. doi:10.1016/j.jadohealth.2007.11.125

Wallace, S. A., Hooper, L. M., \& Persad, M. (2014). Brothers, Sisters and Fictive Kin Communication About Sex Among Urban Black Siblings. Youth \& Society, 46(5), 688-705. doi:10.1177/0044118X12450176

Widman, L., Choukas-Bradley, S., Helms, S. W., Golin, C. E., \& Prinstein, M. J. (2014). Sexual Communication between early adolescents and their dating 
partners, parents, and best friends. The Journal of Sex Research, 51(7), 731741. doi:10.1080/00224499.2013.843148

Widmer, E. D. (1997). Influence of older siblings on initiation of sexual intercourse. Journal of Marriage and Family, 59(4), 928-938. doi:10.2307/353793

Wiederman, M. W. (2005). The gendered nature of sexual scripts. The Family Journal, 13(4), 496-502. doi:10.1177/1066480705278729.

Ybarra, M. L, Emenyonu, N., Nansera, D., Kiwanuka, J., \& Bangsberg, D. R. (2008). Health information seeking among Mbararan adolescents: Results from the Uganda Media and you survey. Health education research, 23(2), 249-258. doi: $10.1093 /$ her/cym026 